\title{
Author Correction to: Moment Generating Functions and Moments of Linear Positive Operators
}

Vijay Gupta, Neha Malik, and Themistocles M. Rassias

\author{
Author Correction to: \\ Chapter 8 in: N. J. Daras, Th. M. Rassias (eds.), \\ Modern Discrete Mathematics and Analysis, \\ Springer Optimization and Its Applications 131, \\ https://doi.org/10.1007/978-3-319-74325-7_8
}

The original version of the chapter was inadvertently published with some errors. The chapter has now been corrected and approved by the authors. 